\title{
Radiologic periodontal findings in paranasal sinus computed tomography scans of chronic rhinosinusitis
} patients*

Sanna Toppila-Salmi'1,2, Anna Julkunen-livari' ${ }^{1}$, Annika Luukkainen', Seija Rhinology Online, vol 3:50-57, 2020 Vento ${ }^{3}$, Satu Apajalahti ${ }^{4}$, Riste Saat ${ }^{4}$, Antti Lehtinen ${ }^{5}$, Jorma Järnstedt ${ }^{5}$, Jura http://doi.org/10.4193/RHINoL/20.033 Numminen ${ }^{6,7}$, Antti Markkola ${ }^{4}$, Heini Huhtala ${ }^{8}$, Ahmed Geneid ${ }^{9}$, Anna Maria Heikkinen ${ }^{10}$, Jukka Meurman ${ }^{10}$

*Received for publication:

April 21, 2020

${ }^{1}$ Haartman Institute, Medicum, University of Helsinki, Helsinki, Finland

${ }^{2}$ Skin and Allergy Hospital, University of Helsinki and Hospital District of Helsinki and Uusimaa, Helsinki University Hospital, Accepted: May 16, 2020

Published: May 25, 2020

Helsinki, Finland

${ }^{3}$ Department of Otorhinolaryngology, University of Helsinki and Hospital District of Helsinki and Uusimaa, Helsinki University Hospital, Helsinki, Finland

${ }^{4}$ Department of Radiology, University of Helsinki and Hospital District of Helsinki and Uusimaa, Helsinki University Hospital, Helsinki, Finland

${ }^{5}$ Medical Imaging Centre, Department of Radiology, Tampere University Hospital, Tampere, Finland

${ }^{6}$ Department of Ear and Oral diseases, Tampere University Hospital, Tampere, Finland

${ }^{7}$ Department of Otorhinolaryngology, Faculty of Medicine and Life Sciences, University of Tampere and Tampere University Hospital, Tampere, Finland

${ }^{8}$ Faculty of Social Sciences, Tampere University, Tampere, Finland

${ }^{9}$ Department of Otorhinolaryngology and Phoniatrics, University of Helsinki and Hospital District of Helsinki and Uusimaa, Helsinki University Hospital, Helsinki, Finland

${ }^{10}$ Department of Oral and Maxillofacial Diseases, University of Helsinki and Hospital District of Helsinki and Uusimaa, Helsinki University Hospital, Helsinki, Finland

\begin{abstract}
Background: Periodontal diseases are a major public health problem with a $70 \%$ prevalence in adults. This retrospective controlled study aimed at studying radiologic periodontal findings in chronic rhinosinusitis with nasal polyps (CRSwNP) and without nasal polyps (sNP).
\end{abstract}

Methodology: Radiologic and patient record data of CRSsNP $(n=66)$ and CRSwNP ( $n=78)$ patients undergoing sinus surgery consultation in 2005-18 were used. Chronic rhinitis/oral disease patients served as a control group ( $n=52)$. Radiologic periodontal findings referring to chronic periodontitis of maxillary premolar and molar region and Lund-Mackay (LM) scores were analyzed blinded from sinus computed tomography (CT) scans.

Results: The prevalence of $\geq 1$ periapical radiolucency was $4.0 \%$ in controls, $14.5 \%$ in CRSsNP and $25.4 \%$ in CRSwNP group, however this difference became insignificant when adjusted by age. In multivariable linear regression model, the presence of $\geq 1$ other radiologic periodontal finding than periapical radiolucency, was significantly associated with high Total LM score. About half (47 $\%$ ) of the sinus CT scans did not visualize cemento-enamel-junction because of limiting radiation.

Conclusions: Radiologic periodontal findings were significantly associated with an increment of Total LM score. Detection of radiologic periodontal findings in CRS patients seems to be possible, if adding the maxillary bone region in sinus CT scans.

Key words: computed tomography, maxillary sinus, nasal polyps, paranasal sinus diseases, periodontitis, rhinitis, sinusitis 


\section{Introduction}

Chronic rhinosinusitis (CRS) is a common, multifactorial and variable disease, where symptom based prevalence has been found to be between $5.5 \%$ and $28 \%{ }^{(1)}$. After failure of medical treatment, the severity of CRS and the putative need for surgery is assessed based on the history, endoscopy, other clinical findings and imaging ${ }^{(1)}$.

\section{Computed tomography (CT) scans are the imaging modality} of choice confirming the extent of pathology and the need for surgery ${ }^{(2)}$. The main findings in CRS are mucosal changes within the osteomeatal complex and/or sinuses. Other characteristic findings of CRS are air-fluid levels, mucosal thickening and opacification of the normally aerated sinus lumen. The only change can be a sclerotic, thickened bone of the sinus wall ${ }^{(3)}$. A number of CT- staging systems of CRS exist; the Lund-Mackay staging is one of the mostly used (4). Staging is based on the degree of opacification ( $0=$ normal, $1=$ partial opacification, $2=$ total opacification) of each sinus: maxillary, anterior ethmoid, posterior ethmoid, sphenoid and frontal sinus, for each side. In addition, the ostiomeatal complex (OMC) is graded as $0=$ not occluded, or $2=$ occluded; resulting a maximum score of 12 per side ${ }^{(4,5)}$.

Periodontitis is a multifactorial, polymicrobial disease of the supporting tissues of the teeth in which the microbiota, genetics and lifestyle factors such as smoking play an important role ${ }^{(6)}$. Periodontal diseases have been reported to affect $70 \%$ of the general population ${ }^{(7-11)}$. Chronic periodontal disease is associated with cardio- and cerebrovascular diseases ${ }^{(12,13)}$, diabetes mellitus ${ }^{(14,15)}$, cancer ${ }^{(16,17)}$, odontogenic sinusitis ${ }^{(18)}$, and CRS ${ }^{(19)}$.

Radiologic diagnostics of periodontitis include tooth loss, increased distance between the cemento-enamel junction and alveolar bone, vertical bone loss and periapical radiolucency (20). Dental pathologies have been shown to be common in a population that had undergone sinus CT scans due to suspicion of CRS, trauma or tumor ${ }^{(21)}$. Periodontitis has been shown to associate with radiologic signs of sinusitis ${ }^{(22)}$. Periapical lesions have shown to be associated with radiologic maxillary sinus mucosal thickening ${ }^{(23)}$. Increased prevalence of radiological maxillary mucosal chronic thickenings and mucous cysts have been detected in dentate patients aged over 50 years, compared with edentate controls of the same age group ${ }^{(24)}$.

Odontogenic etiology has been detected to be part of recurrent acute rhinosinusitis (ARS) ${ }^{(1)}$. Dental infection has been linked to difficult-to-treat CRS in an interview survey of rhinologists ${ }^{(1)}$.
A retrospective study of Finnish tertiary care patients with acute rhinosinusitis showed that the proportion of patients with odontogenic nature of acute rhinosinusitis was $15 \%{ }^{(25)}$.

Although CRSwNP and chronic periodontitis have both high prevalence, there is little knowledge of the potential comorbidity between CRSWNP and chronic periodontitis in general population. Literature exists of odotogenic ARS and the anatomical connection between odontogenic sinusitis and periapical lesions, however, knowledge is lacking of the association between radiologic periodontal findings referring to chronic periodontitis and CRS in general. We therefore aimed at studying radiologic periodontal findings referring to chronic periodontitis in CRSsNP, CRSWNP and control patients visiting tertiary care and having the sinus CT scans taken for medical purposes. The hypothesis was that the radiologic periodontitis findings are associated with CRSwNP, oral disease and high LM score.

\section{Materials and Methods}

\section{Subjects}

This retrospective cross-sectional study was carried out at Tampere and Helsinki University Hospitals. The study was approved by the ethical committee of the Hospital Districts (nro 31/13/03/00/2015). Ethics committee provided an approval that there was no need for written informed consent for this retrospective study.

Data of a total of 66 CRSsNP and 78 CRSwNP patients, and 52 control patients undergoing surgical consultation at the Department of Otorhinolaryngology or Oral and Maxillofacial Surgery between 2005 and 2017 were used. Subjects in each group were randomly selected. Patients were without patient record evidence of periodontitis. Inclusion criteria were age > 16 years, sinus CT scan data and patient record data available at time of consultation, including information of endoscopic nasal polyps. Exclusion criteria were surgery 12 months prior to $C T$ scans, acute rhinosinusitis or trauma at the time of CT scans, a history of chronic periodontitis, severe disease (such as active cancer), fungal rhinosinusitis, cystic fibrosis, and primary ciliary dyskinesia.

\section{Outcomes}

Outcomes were the phenotype and total LM score of sinus CT scans. CRSsNP and CRSwNP phenotypes were defined according to the European Position Paper on CRS and nasal polyps (1), and by a $L M$ score of $\geq 1 / 24$. Control patients underwent routine sinus $\mathrm{CT}$ scans due to consideration of maxillofacial (such as orthognathic) surgery $(n=42)$ or chronic rhinitis $(n=10)$, 

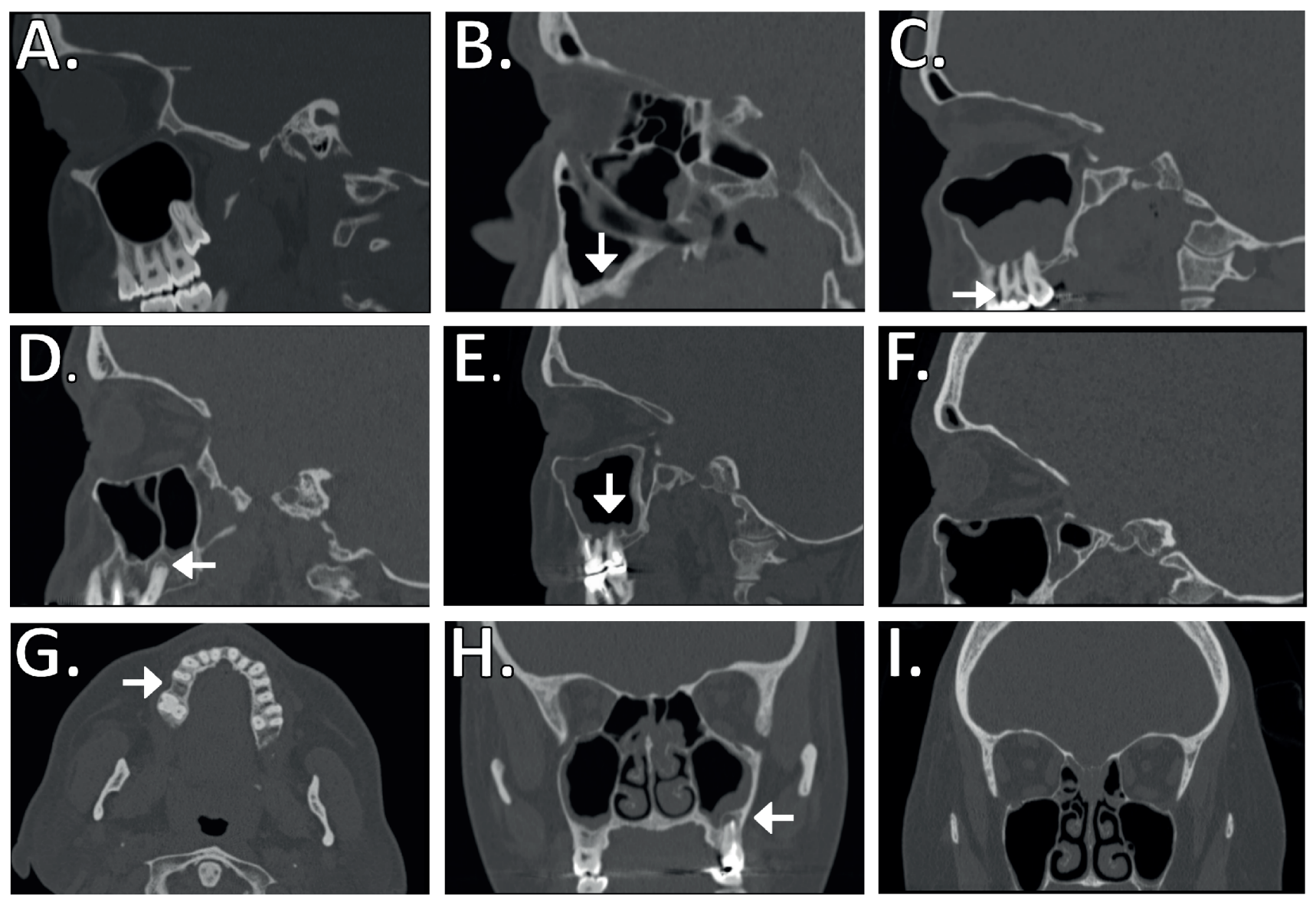

Figure 1. Radiologic periodontal finding(s) in maxillary premolar/molar region of sinus CT scans. Arrows indicate the radiologic periodontal signs, which are periapical radiolucency, vertical bone loss, and $\geq 2 \mathrm{~mm}$ distance between cemento-enamel junction and alveolar bone. Although in the Figure only one $\mathrm{CT}$ slice of one projection has been showed per each example case, the radiologic periodontal signs and LM scores were assessed from all projections and slices. A-F. in sagittal view; G. in axial view; H-L. in coronal view. A. No radiologic signs of periodontitis. Maxillary sinus Lund Mackay score $\left(L_{m}\right.$ max $)$ is 0 . B. Vertical alveolar bone loss. $L M_{\max }=1$. C. Increased distance between cemento-enamel junction and alveolar bone (over 4 $\mathrm{mm}) . \mathrm{LM}_{\max }=1$. D. Periapical radiolucency not in contact with maxillary sinus. $\mathrm{LM}_{\max }=1$. E. Periapical radiolucency in contact with maxillary sinus mucosa $L M_{\max }=1$. F. Not detectable maxillary alveolar bone region due to limiting radiation purposes. $L_{M} M_{\max }=1$. G. Vertical alveolar bone loss. $\mathrm{H}$. Periapical radiolucency in contact with maxillary sinus mucosa $\mathrm{LM}_{\max }=1$. I. Not detectable maxillary alveolar bone region due to limiting radiation purposes. $L M_{\max }=1$. Total $L M$ score is scored $0=$ no opacification $1=$ partial opacification $2=$ complete opacification of the frontal, anterior ethmoidal, posterior ethmoidal, sphenoidal sinuses, plus osteomeatal complex ( $0=$ not obstructed, $2=$ obstructed $)$.

and all controls were without patient record evidence of CRS or periodontitis. The controls who had rhinitis were using adequately with nasal medication prior to sinus CT scans. In addition control chronic rhinitis patients had a Total LM score $=0$. Total LM score is scored $0=$ no opacification $1=$ partial opacification $2=$ complete opacification of the frontal, anterior ethmoidal, posterior ethmoidal, sphenoidal sinuses, plus osteomeatal complex $(0=$ not obstructed, $2=$ obstructed), respectively from both sides.

\section{Variables of interest $=$ Radiologic periodontal findings} CT-scans were performed using 16-row CT scans of GE Medical Healthcare (Chicago, IL, USA), Siemens Somatom (Munich, Germany) or Toshiba Aquilion (Tokyo, Japan), and 64-row CT scans of Philips (Best, Netherlands) ${ }^{(26,27)}$. Sinus CT scans were observed by independent observers blinded to each other and to patient history data. LM scores were evaluated by head and neck radiologist (AM) and otorhinolaryngologist (STS). Maxillary region was evaluated by three dentomaxillofacial radiologists ( $\mathrm{JJ}, \mathrm{AL}$ and SA) blinded to each other. In situations of disagreement, the structures were re-evaluated and a consensus was found. Five radiologic periodontal signs were evaluated from premolarmolar region of the upper jaw per each side:

- $\quad$ periapical radiolucency ( 0 vs. $\geq 1$ )

- $\quad$ vertical bone loss (0 vs. $\geq 1$ )

- $\quad \geq 2 \mathrm{~mm}$ distance between cemento-enamel junction and alveolar bone ( 0 vs. $\geq 1$ )

- $\quad$ toothless upper jaw (no vs. yes)

- $\quad$ difficulties to visualize (no vs. yes; reason if yes) 
Table 1. Comparison of baseline characteristics and radiologic periodontal findings between control patients, CRSsNP and CRSwNP groups.

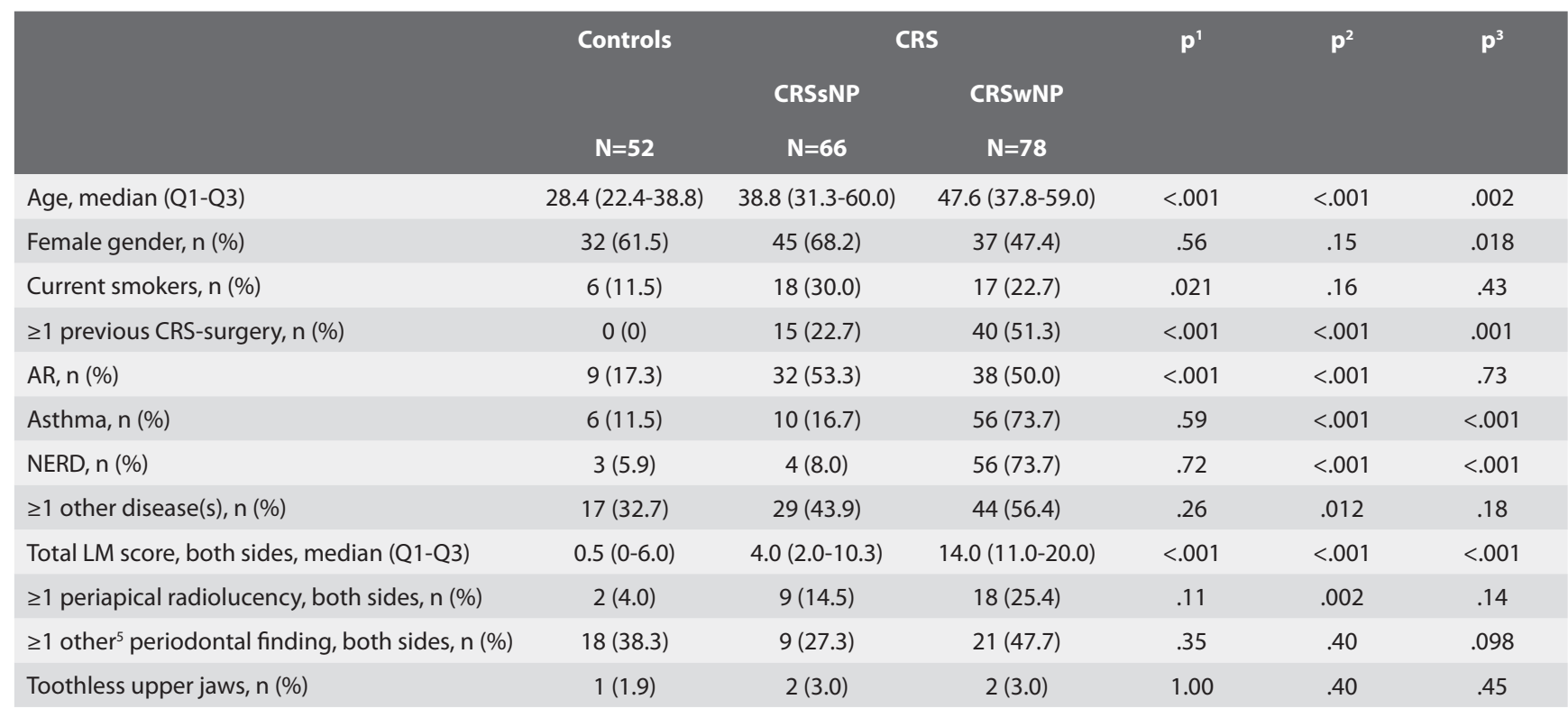

$\mathrm{CRS}=$ chronic rhinosinusitis; $\mathrm{AR}=$ allergic rhinitis; $\mathrm{NP}=$ nasal polyps; $\mathrm{NERD}=$ patient-reported aspirin exacerbated respiratory disease. $\mathrm{P}$ values by Fisher's exact test (dichotomous variables) or Kruskal Wallis and Mann Whitney U test (continuous variables). Q1 $=25 \%$ percentile, Q3 $=75 \%$ percentile. ' $p$-value by Control vs. CRSsNP group. ${ }^{2} p$-value by Control vs. CRSwNP group. ${ }^{3} p$-value by CRSsNP vs. CRSwNP group. ${ }^{4} \mathrm{Chronic}$ rhinitis/Oral disease patients who underwent routine sinus $\mathrm{CT}$ scans. ${ }^{5}$ Other periodontal finding means vertical bone loss AND/OR $\geq 2$ mm distance between and alveolar bone and cemento-enamel junction, the latter was visualized only in 67 (46.5\%) patients due to limiting radiation purposes. \% values counted from complete cases. Total LM score is scored $0=$ no opacification $1=$ partial opacification $2=$ complete opacification of the frontal, anterior ethmoidal, posterior ethmoidal, sphenoidal sinuses, plus osteomeatal complex ( $0=$ not obstructed, $2=0$ obstructed).

Two main variables of interest were used: presence of $\geq 1$ periapical radiolucency in both sides, and $\geq 1$ other periodontal finding in both sides, in which the "other periodontal finding" was defined as the presence of vertical bone loss and/or $\geq 2 \mathrm{~mm}$ distance between cemento-enamel junction and alveolar bone.

\section{Covariates}

Eight covariates were selected based on the literature and data available: gender, age, smoking (no vs. current), previous CRSsurgery (no vs. $\geq 1$ ), allergic rhinitis (no vs. yes), asthma (no vs. yes), NERD (no vs. yes), any other general disease (no vs. yes). Asthma and/or NERD -covariate was used in regression models. Asthma, NERD and AR diagnoses were based on self-reported doctor diagnosed diseases. In addition to typical symptoms, a doctor-diagnosed asthma is based on lung-function test results, a doctor-diagnosed. AR is based on positive skin prick test or serum specific lgE results. NERD diagnosis is based on a positive history of wheeze/cough or naso-ocular symptoms after intake of NSAID.

\section{Statistical analysis}

Statistical analysis was carried out by the SPSS Base 15.0 Statistical Software Package (SPSS Inc., Chicago, IL, USA). The associati- ons between each risk factor and severe asthma were estimated using Fisher's exact test (dichotomous), Mann Whitney $U$ test (continuous), and by using binary logistic- and linear regression models. Odds ratios (OR) with 95\% confidence intervals are reported. Factors associated statistically significantly with LM score, were included in a multiple regression model. A twotailed value of $p<0.05$ was considered statistically significant.

\section{Results}

Radiologic signs of periodontitis in the three patient groups Patient characteristics are given in Table 1. The mean (min-max) age of the subjects was 41.9 (17.3-74.4) years (Table 1). Of the patients, 114 (58.2\%) were women, 41 (20.9\%) were current smokers, 79 (40.3\%) had allergic rhinitis (AR), 72 (36.7\%) had asthma, and 49 (25.0\%) had NERD. None of the control patients and 55 (38.2\%) of the CRS patients had undergone previous CRS-surgery $(p<0.001)$. Smoking was the most prevalent $(30.0$ \%) in CRSsNP group (Table 1). Compared to controls, CRSsNP and CRSwNP patients were statistically significantly older, had higher total LM score, and had more AR. CRSwNP group had the highest median age and presented more with co-morbidities (asthma, NERD or other diseases, Table 1).

The prevalence of $\geq 1$ periapical radiolucency was $4.0 \%$ in con- 
Table 2. Association of radiologic periodontal findings and seven covariates with the Total Lund-Mackay (LM) score (0-24, from both sides) of sinus computed tomography scans in all patients.

\begin{tabular}{|c|c|c|c|c|c|c|c|c|c|c|}
\hline & & n & $\begin{array}{l}\text { Median } \\
\text { LM Score }\end{array}$ & IQR & $\boldsymbol{\beta}$ & $\begin{array}{l}\text { Model } \\
95 \% \mathrm{Cl}\end{array}$ & $p_{1}$ & $\beta$ & $\begin{array}{l}\text { Model }_{2} \\
95 \% \mathrm{Cl}\end{array}$ & $\mathbf{p}_{2}$ \\
\hline $\begin{array}{l}\geq 1 \text { periapical radiolu- } \\
\text { cency, both sides }\end{array}$ & $\begin{array}{l}\text { No } \\
\text { Yes }\end{array}$ & $\begin{array}{c}154 \\
29\end{array}$ & $\begin{array}{c}7.0 \\
12.0\end{array}$ & $\begin{array}{l}2.0-13.0 \\
8.0-14.5\end{array}$ & $\begin{array}{c}\text { ref } \\
0.19\end{array}$ & $0.87-6.32$ & .010 & $\begin{array}{c}\text { ref } \\
-0.03\end{array}$ & $-3.33-2.19$ & .68 \\
\hline $\begin{array}{l}\geq 1 \text { other }^{1} \text { periodontal } \\
\text { finding, both sides }\end{array}$ & $\begin{array}{l}\text { No } \\
\text { Yes }\end{array}$ & $\begin{array}{l}76 \\
48\end{array}$ & $\begin{array}{c}5.5 \\
11.5\end{array}$ & $\begin{array}{l}1.0-11.8 \\
2.5-16.8\end{array}$ & $\begin{array}{c}\text { ref } \\
0.25\end{array}$ & $1.08-5.90$ & .005 & $\begin{array}{c}\text { ref } \\
0.22\end{array}$ & $0.93-5.16$ & .005 \\
\hline Group & $\begin{array}{l}\text { Control } \\
\text { CRS }^{3}\end{array}$ & $\begin{array}{c}52 \\
144\end{array}$ & $\begin{array}{c}0.5 \\
11.0\end{array}$ & $\begin{array}{c}0-6.0 \\
4.0-14.8\end{array}$ & $\begin{array}{c}\text { ref } \\
0.48\end{array}$ & $5.57-9.47$ & $<.001$ & $\begin{array}{c}\text { ref } \\
0.44\end{array}$ & $3.79-8.43$ & $<.001$ \\
\hline Age & & & & & 0.22 & $0.04-0.17$ & .002 & -0.06 & $-0.10-0.05$ & .45 \\
\hline Male gender & $\begin{array}{l}\text { No } \\
\text { Yes }\end{array}$ & $\begin{array}{l}114 \\
82\end{array}$ & $\begin{array}{c}6.5 \\
10.5\end{array}$ & $\begin{array}{l}1.8-13.0 \\
5.0-15.3\end{array}$ & $\begin{array}{l}\text { ref } \\
0.21\end{array}$ & $1.05-4.94$ & .003 & $\begin{array}{c}\text { ref } \\
0.08\end{array}$ & $-0.80-3.06$ & .25 \\
\hline Smoking & $\begin{array}{l}\text { No } \\
\text { Yes }\end{array}$ & $\begin{array}{c}146 \\
41\end{array}$ & $\begin{array}{c}8.0 \\
11.0\end{array}$ & $\begin{array}{l}2.0-14.0 \\
3.0-14.0\end{array}$ & $\begin{array}{l}\text { ref } \\
0.06\end{array}$ & $-1.43-3.45$ & .42 & & $\begin{array}{c}\text { not } \\
\text { entered }\end{array}$ & \\
\hline AR & $\begin{array}{l}\text { No } \\
\text { Yes }\end{array}$ & $\begin{array}{c}109 \\
79\end{array}$ & $\begin{array}{c}7.0 \\
12.0\end{array}$ & $\begin{array}{l}2.0-13.0 \\
3.0-14.0\end{array}$ & $\begin{array}{l}\text { ref } \\
0.18\end{array}$ & $0.54-4.55$ & .013 & $\begin{array}{c}\text { ref } \\
0.03\end{array}$ & $-1.66-2.53$ & .68 \\
\hline Asthma and/or NERD & $\begin{array}{l}\text { No } \\
\text { Yes }\end{array}$ & $\begin{array}{c}112 \\
76\end{array}$ & $\begin{array}{c}5.0 \\
14.0\end{array}$ & $\begin{array}{l}1.0-11.0 \\
9.3-19.8\end{array}$ & $\begin{array}{c}\text { ref } \\
0.53\end{array}$ & $5.76-9.24$ & $<.001$ & $\begin{array}{c}\text { ref } \\
0.34\end{array}$ & $2.53-6.92$ & $<.001$ \\
\hline$\geq 1$ other disease(s) & $\begin{array}{l}\text { No } \\
\text { Yes }\end{array}$ & $\begin{array}{l}76 \\
48\end{array}$ & $\begin{array}{c}5.5 \\
11.5\end{array}$ & $\begin{array}{l}1.0-11.8 \\
2.5-16.8\end{array}$ & $\begin{array}{c}\text { ref } \\
0.13\end{array}$ & $-0.12-3.78$ & .067 & & $\begin{array}{c}\text { not } \\
\text { entered }\end{array}$ & \\
\hline
\end{tabular}

Model $1=$ Univariate analysis. Model $2=$ Multivariable analysis by the variable that were associated at $\mathrm{p}<0.05$ level in the Model 1. CRS $=$ chronic rhinosinusitis; $\mathrm{AR}=$ allergic rhinitis; $\mathrm{NP}=$ nasal polyps; $\mathrm{NERD}=$ patient-reported aspirin exacerbated respiratory disease. $\mathrm{IQR}=\mathrm{interquartile}$ range $(25$ th percentile - 75th percentile), $\beta=$ standardized beta coefficient; P-values by linear regression model. 'Other periodontal finding means vertical bone loss AND/OR $\geq 2 \mathrm{~mm}$ distance between and alveolar bone and cemento-enamel junction, the latter was visualized only in 67 (46.5\%) patients due to limiting radiation purposes. \% values counted from complete cases. ${ }^{2}$ Chronic rhinitis/Oral disease patients who underwent routine sinus CT scans. ${ }^{3}$ CRSsNP or CRSwNP group. Total LM score is scored $0=$ no opacification $1=$ partial opacification $2=$ complete opacification of the frontal, anterior ethmoidal, posterior ethmoidal, sphenoidal sinuses, plus osteomeatal complex ( $0=$ not obstructed, $2=$ obstructed).

trols, $14.5 \%$ in CRSsNP and $25.4 \%$ in CRSwNP group ( $p=0.004$, Table 1). The prevalence of $\geq 1$ periapical radiolucency that continued into the bottom maxillary sinus was $0 \%$ in controls, $11.3 \%$ in CRSsNP and $14.1 \%$ in CRSwNP group $(p=0.010)$. Compared to controls, CRSwNP patients had higher prevalence of $\geq 1$ periapical radiolucency ( $p=0.002$, Table 1 ). When entering age and $\geq 1$ periapical radiolucency in a multivariable model-1, CRSwNP was significantly associated with higher age (OR [CI95\%] $=1.08$ [1.04-1.11], $\mathrm{p}<0.001)$, and by an insignificant trend with $\geq 1$ periapical radiolucency (4.60 [0.91-23.3], $p=0.066)$, when having the controls as a reference group. When entering smoking, age and $\geq 1$ periapical radiolucency in a multivariable model-2, CRSwNP was still significantly associated with higher age (1.08 [1.04-1.11], $\mathrm{p}<0.001$ ), by an insignificant trend with $\geq 1$ periapical radiolucency (4.14 [0.80-21.33], $\mathrm{p}=0.089)$, and not significantly with smoking (1.87 [0.56-6.19], $\mathrm{p}=0.31)$, when having the controls as a reference group.

Over all the prevalence of $\geq 1$ other periodontal finding than periapical radiolucency, was 48 (24.5\%), however this was visualized only in 67 (46.5\%) of patients due to radiation minimizing purposes. At least one other periodontal finding was not sta- tistically significantly associated with any patient group (Table 1). Of these signs, the prevalence of $\geq 1$ vertical bone loss was $17.0 \%$ in controls, $15.2 \%$ in CRSsNP and $20.5 \%$ in CRSwNP group $(p=0.87$ ). The prevalence of one or more findings of $\geq 2 \mathrm{~mm}$ distance between cemento-enamel junction and alveolar bone was $38.3 \%$ in controls, $18.8 \%$ in CRSsNP and $41.9 \%$ in CRSwNP group ( $p=0.085$ ). The prevalence of toothless upper jaw was 1 (1.9\%) in controls, 2 (3.0\%) in CRSsNP and 5 (6.4\%) in CRSwNP group $(p=0.48)$.

Radiologic signs of periodontitis and its association with the Total LM score

In univariate model presence of $\geq 1$ periapical radiolucency was statistically significantly associated with increased Total LM score reflecting mucosal thickening of paranasal sinuses and maxillary sinus ostia (beta coefficient, $\mathrm{t}[\mathrm{Cl} 95 \%]=0.19,2.60$ [0.87-6.32], $p=0.010$, Table 2). Similarly, presence of $\geq 1$ other periodontal finding was statistically significantly associated with increased Total LM score $(0.25,2.87$ [1.08-5.90], $p=0.005$, Table 2$)$. When entering these two radiologic periodontitis signs and seven covariates into the multivariable model, the presence of $\geq 1$ other periodontal finding, CRS or asthma/NERD were all significantly 
associated with increased Total LM score $(p<0.006$, Table 2$)$.

\section{Discussion}

Periodontital diseases affect $70 \%$ of the global population, with variations between developing and developed countries ${ }^{(7-11)}$. In a Taiwanese population study, periodontitis had an incidence rate of 5.12 per 100 person years in CRS population in contrast to an incidence rate of $3.24 \%$ in the control population ${ }^{(19)}$. In our study, CRSwNP was associated with increased prevalence of periapical radiolucency, as compared with controls. Yet due to the higher median age of CRSwNP group, the difference became insignificant trend when adjusted with age, whereas smoking did not remarkably affect the trend. In addition radiologic periodontal findings were significantly associated with increased total LM score when adjusted by covariates, such as age. To our knowledge this is the first preliminary study suggesting that the inflammation in one area namely periodontitis drives inflammation throughout the sinuses. It may also indicate that there might be a shared inflammatory mechanism between CRS(wNP) and chronic periodontitis in general, and not only an anatomical connection. Yet it is also possible that nasal obstruction and mouth breathing of CRS patients increase the risk of chronic periodontitis. Thus, this putative co-morbidity needs to be confirmed in general population, with age-matched groups. Also prospective follow-up studies are needed including endoscopic nasal polyp scoring, and clinical diagnostics of periodontitis of all teeth.

To our knowledge, there is little previous evidence of the prevalence of radiologic periodontal findings when evaluating routinely taken sinus CT scans. Radiologic periodontal findings have shown to be tooth loss, increased distance between cemento-enamel junction and alveolar bone, vertical bone loss and periapical radiolucency ${ }^{(20)}$. Previously, Brullman et al. detected a pronounced association between periodontitis and radiological signs of sinusitis ${ }^{(22)}$. Shanbhag et al. found an association between the maxillary sinus mucosal thickening and periapical lesions ${ }^{(23)}$. Moreover, polypoid type thickening was frequently observed when the periapical lesion is in contact with or entered the sinus floor ${ }^{(23)}$, which is partly in line with our findings. Interestingly, another study group showed that dental pathologies were encountered in more than half of the patients, in a population that had undergone sinus $\mathrm{CT}$ scans due to suspicion of CRS, trauma or tumor ${ }^{(21)}$. Berkun et al. detected that mean number of missing posterior teeth correlated with postnasal drip and nasal congestion, and that periapical pathology was associated with nasal discharge or runny nose in a group of 81 asymptomatic subjects ${ }^{(28)}$. This result is similar to our findings of the relatively high prevalence (5\%) of toothless upper jaw in CRS patients. In epidemiologic studies loss of teeth has shown to correspond well to periodontitis ${ }^{(29,30)}$. These findings highlight the need for primary care doctors to assess oral health, including detection of tooth loss, and to suspect periodontitis or sinonasal morbidities. The other comorbidities such as cardiovascular diseases, especially in risk groups should neither be forgotten. On the other hand, dentists could inform his/her patients with periodontitis that they might also have an increased risk of CRSWNP.

Despite their high prevalence, there is little knowledge of the potential comorbidity between CRSwNP and chronic periodontitis in general population. Although this study was not population-based, the results could indicate that chronic periodontitis could be an independent risk factor for the development of CRSwNP. On the other hand chronic periodontitis is associated with higher age and with other diseases. Due to higher median age of the CRSWNP group compared to control group, and the cross sectional nature of our study, we were not able to confirm an association or a causality between these two diseases. It is possible that CRS patients have nasal obstruction and mouth breathing, which may predispose to development/aggravation of chronic periodontitis either alone or together with other in part unknown factors. In the future, the putative comorbidity would be important to know for assessing diagnosis, disease severity, prognosis, and optimal treatment plan as well as management with appropriate health care professionals. There is a known link between periodontitis and diabetes, CVDs, cancer, or other general diseases. Thus it might be important to inform CRS patients at a risk about the association of uncontrolled periodontitis with other chronic diseases, such as cardiovascular diseases, diabetes mellitus, autoimmune diseases and cancer $(10-12,14-17,29,31)$. In the future, a follow-up study would be warranted to evaluate whether periodontitis indeed affects CRSwNP and its progression and/or vice versa. A genome-microbiome interaction study would also be warranted to study whether there are shared inflammatory mechanisms between periodontitis and CRSwNP, as could be supposed based on our findings. Osteitis may provide a link between CRS and chronic periodontitis and would be worth of future investigations.

Our study population consisted of a random sample of subjects who underwent routine sinus CT scans due to suspicion of CRS or due to oral disease. Thus our study was able to demonstrate a larger connection between radiologic periodontal findings in CRS-subjects. We found that any radiologic periodontal finding is associated with CRSwNP phenotype, not only with odontogenic sinusitis cases. Previous studies have shown an association between periapical dental lesions and odotogenic sinusitis, which have been supposed to be due to anatomical connection between the premolar-molar region of upper jaw and maxillary sinus ${ }^{(18)}$. In our study, the prevalence of radiologic signs of periapical radiolucency with maxillary sinus signs was $11 \%$ 
in the CRSwNP group, $3 \%$ in the CRSsNP group and $0 \%$ in the rhinitis group. Compared to the literature ${ }^{(18)}$, our prevalence values were lower, however this could be explained by our lacking clinical dental data.

We found that there seems to be considerable variation in the performance quality of sinus CT scans to recognize the alveolar bone region of the maxilla. In almost half of the sinus CT scans, the upper jaw region was not properly visualized, probably due to limiting the radiation exposure. We did not want to leave out the cases with non-visualized upper jaw, as visualizing difficulty and its reason, was one variable of our interests. The putative co-morbid periodontitis and CRSwNP, that was found, stresses the importance that a radiologist performing sinus CT scans should routinely make sure that also the upper jaw region is visualized in every sinusitis case.

We acknowledge that there was a lack of clinical oral health data with periodontal health parameters, nasal endoscopic polyp score data, and follow-up data. The sample was small and not population-based, and the control and CRS groups differed by age, which limited the possibility to evaluate association between periapical radiolucency and CRSwNP in multivariable models. Due to ethical reasons and the retrospective nature of the study, we were not able to perform sinus CT scans of healthy controls, and thus non-CRS patients with oral disease/AR served as a control group, which may affect in part the results. We also acknowledge that slight residual confounding effect of smoking might remain due to lack of smoking information of some subjects.

\section{Conclusions}

Radiologic periodontal findings were significantly associated with an increment of Total LM score. The clinical relevance of the finding needs to be confirmed by prospective controlled follow-up studies including clinical periodontal diagnostics. Our findings also stress the importance that sinus CT scans of
CRS patients should always be performed so that the upper jaw region is visualized.

\section{Acknowledgments}

The authors thank research nurse Marja-Leena Oksanen for excellent assistance. The study was supported in part by research grant of Aatos Erkko Foundation, Tampere Tuberculosis association, the Finnish Medical Society Duodecim,, the Medical Society of Finland, the Finnish Society of Allergology and Immunology, State funding for university-level health research (TYH2018103, TYH2019322), the Väinö and Laina Kivi Foundation, the Yrjö Jahnsson Foundation.

\section{Authorship contribution}

ST-S, AJ, SA, JJ, AL, AM, JN and SV provided the study plan, made the applications, collected the data and evaluated the CT scans. ST-S and HH performed the data analyses. ST-S, AJ, $\mathrm{AL}, \mathrm{AMH}$ and JM wrote the manuscript. All authors reviewed critically the manuscript.

\section{Conflict of interest}

STS has acted as paid consultant for ERT, Novartis, Roche Products and Sanofi Pharma. All these are outside the submitted work. All other authors declare no conflicts of interest.

\section{Ethics approval and consent to participate}

Not applicable.

\section{Consent for publication}

Not applicable.

\section{Availability of data and materials}

Not applicable.

\section{Funding}

Not applicable.

\section{References}

1. Fokkens WJ, Lund VJ, Hopkins C, Hellings PW, Kern R, Reitsma S, et al. European Position Paper on Rhinosinusitis and Nasal Polyps 2020. Rhinology. 2020. 20;58(Suppl S29):1-464

2. Holbrook EH, Brown CL, Lyden ER, Leopold DA. Lack of significant correlation between rhinosinusitis symptoms and specific regions of sinus computer tomography scans. Am J Rhinol. 2005;19(4):382-7.

3. Fokkens WJ, Lund VJ, Mullol J, Bachert C, Alobid I, Baroody F, et al. EPOS 2012 European position paper on rhinosinusitis and nasal polyps 2012. A summary for otorhinolaryngologists. Rhinology

\section{2;50(1):1-12.}

4. Lund VJ, Kennedy DW. Staging for rhinosinusitis. Otolaryngol - Head Neck Surg [Internet]. 1997;117(3):S35-40.

5. Hopkins C, Browne JP, Slack R, Lund V, Brown P. The Lund-Mackay staging system for chronic rhinosinusitis: how is it used and what does it predict? Otolaryngol Head Neck Surg. 2007/10/02. 2007;137(4):555-61.

6. Stabholz A, Soskolne WA, Shapira L. Genetic and environmental risk factors for chronic periodontitis and aggressive periodontitis. Periodontol 2000. 2010 Jun;53:138-53.

7. Feres M, Teles F, Teles R, Figueiredo LC, Faveri M. The subgingival periodontal microbiota of the aging mouth. Periodontol
2000. 2016 Oct;72(1):30-53.

8. Wahlin A, Papias A, Jansson $H$, Norderyd O. Secular trends over 40 years of periodontal health and disease in individuals aged 20-80 years in Jonkoping, Sweden: Repeated cross-sectional studies. J Clin Periodontol. 2018 Sep;45(9):1016-24

9. Kassebaum NJ, Bernabe E, Dahiya M Bhandari B, Murray CJL, Marcenes W. Global burden of severe periodontitis in 19902010: a systematic review and meta-regression. J Dent Res. 2014 Nov;93(11):1045-53.

10. Raitapuro-Murray T, Molleson TI, Hughes FJ. The prevalence of periodontal disease in a Romano-British population c. 200-400 AD. Br Dent J. 2014 Oct;217(8):459-66. 
11. Sheiham A. Oral health, general health and quality of life. Vol. 83, Bulletin of the World Health Organization. 2005. p. 644

12. Meurman JH, Sanz M, Janket S-J. Ora health, atherosclerosis, and cardiovascular disease. Crit Rev Oral Biol Med. 2004 Nov;15(6):403-13.

13. Campanella V, Oberti L, Gabrione F, Gonzalez-Valero L, Hernandez-Martinez V, Silvestre-Rangil J. Periodontitis and cerebrovascular disease: a new novel in medicine. J Biol Regul Homeost Agents. 2019;33(3 Suppl. 1):135-44.

14. Meurman JH, Bascones-Martinez A. Are oral and dental diseases linked to cancer? Oral Dis. 2011 Nov;17(8):779-84.

15. Preshaw PM, Alba AL, Herrera $D$, Jepsen S, Konstantinidis A, Makrilakis K, et al. Periodontitis and diabetes: a two-way relationship. Diabetologia. 2012 Jan;55(1):2131.

16. Zhang Y, He J, He B, Huang R, Li M. Effect of tobacco on periodontal disease and oral cancer. Tob Induc Dis. 2019;17:40.

17. Shao J, Wu L, Leng W-D, Fang C, Zhu Y-J, Jin $\mathrm{Y}-\mathrm{H}$, et al. Periodontal Disease and Breast Cancer: A Meta-Analysis of 1,73,162 Participants. Front Oncol. 2018;8:601.

18. Little RE, Long CM, Loehrl TA, Poetker DM Odontogenic sinusitis: A review of the current literature. Laryngoscope Investig Otolaryngol. 2018 Apr;3(2):110-4.

19. Keller JJ, Wu C-S, Lin H-C. Chronic rhinosinusitis increased the risk of chronic periodontitis: a population-based matchedcohort study. Laryngoscope. 2013 Jun;123(6):1323-7.

20. Aljehani YA. Diagnostic Applications of Cone-Beam CT for Periodontal Diseases. Int
J Dent. 2014;2014:865079

21. Bulbul E, Yanik B, Demirpolat G. Detection of Dental Pathologies in Routine Paranasal CT Scans: A Retrospective Study. J Clin Diagn Res. 2017 Jul;11(7):TC17-20.

22. Brullmann DD, Schmidtmann I, Hornstein S, Schulze RK. Correlation of cone beam computed tomography (CBCT) findings in the maxillary sinus with dental diagnoses: a retrospective cross-sectional study. Clin Oral Investig. 2012 Aug;16(4):1023-9.

23. Shanbhag S, Karnik P, Shirke P, Shanbhag $\checkmark$. Association between periapical lesions and maxillary sinus mucosal thickening: a retrospective cone-beam computed tomographic study. J Endod. 2013 Jul;39(7):853-

24. Mathew AL, Pai KM, Sholapurkar AA Maxillary sinus findings in the elderly: a panoramic radiographic study. J Contemp Dent Pr. 2009/12/19. 2009;10(6):E041-8.

25. Wuokko-Landén A, Blomgren K, Välimaa $H$ Acute rhinosinusitis - are we forgetting the possibility of a dental origin? A retrospective study of 385 patients. Acta Otolaryngol 2019 Sep;139(9):783-7.

26. Julkunen A, Terna $E$, Numminen J Markkola A, Dastidar P, Karjalainen M, et al. Inter-observer agreement of paranasal sinus computed tomography scans. Acta Otolaryngol. 2017 Jun;137(6):611-617.

27. Karjalainen M, Julkunen A, Markkola A, Prasun D, Huhtala H, Mikko S, et al. Reproducibility of $3 \mathrm{~mm}$-Slice-Thick Reconstruction of Paranasal Sinus Computed Tomography Scans. Open $J$ Radiol. 2016, 6, 39-48.

28. Horwitz Berkun R, Polak D, Shapira L, Eliashar R. Association of dental and max- illary sinus pathologies with ear, nose, and throat symptoms. Oral Dis. 2018 May;24(4):650-6.

29. Julkunen A, Heikkinen AM, Söder B, Söder P-Ö, Toppila-Salmi SK, Meurman JH. Autoimmune Diseases and Oral Health: 30-Year Follow-Up of a Swedish Cohort. Dent J. 2017 Dec 22;6(1):1

30. Nibali L, Farias BC, Vajgel A, Tu YK, Donos $N$. Tooth loss in aggressive periodontitis: a systematic review. J Dent Res. 2013 Oct;92(10):868-75.

31. Locker D, Slade GD, Murray H. Epidemiology of periodontal disease among older adults: a review. Periodontol 2000. 1998 Feb;16:1633.

Sanna Toppila-Salmi, MD PhD

Skin and Allergy Hospital

Helsinki University Hospital and

University of Helsinki

PO Box 160 (Meilahdentie 2)

Fl 00029 Hospital District of Helsinki

and Uusimaa

Helsinki

Finland

Tel +358505431421

Fax +358 294126382

E-mail: sanna.salmi@helsinki.fi

ORCID: 0000-0003-0890-6686

ISSN: 2589-5613 / @2020 The Author(s). This work is licensed under a Creative Commons Attribution 4.0 International License. The images or other third party material in this article are included in the article's Creative Commons license, unless indicated otherwise in the credit line; if the material is not included under the Creative Commons license, users will need to obtain permission from the license holder to reproduce the material. To view a copy of this license, visit http://creativecommons.org/ licenses/by/4.0/ 